\title{
Maternal and Neonatal Outcomes during Dengue Infection Outbreak at a Tertiary National Hospital in Endemic Area of Indonesia
}

\author{
Ryan Saktika Mulyana', Evert Solomon Pangkahila', Tjokorda Gede Astawa Pemayun ${ }^{2, *}$ \\ ${ }^{1}$ Maternal-Fetal Medicine Division, Obstetrics and Gynecology Department, Udayana University and Sanglah Hospital, Denpasar, Indonesia \\ ${ }^{2}$ Obstetrics and Gynecology Residency, Obstetrics and Gynecology Department, Udayana University and Sanglah Hospital, Denpasar, Indonesia
}

Background: Dengue infection is the most common mosquito-borne disease in Indonesia. Dengue incidence during pregnancy increases with its prevalence among women of reproductive ages. It potentially causes serious maternal and neonatal morbidity and mortality; however, its impact during a period of disease outbreak has not been described. We aimed to assess the outcomes of pregnancies affected by dengue infection during an outbreak in an endemic area of Indonesia.

Methods: We conducted a prospective observational study at Sanglah General Hospital, Bali, Indonesia from April 2016 to April 2017. All pregnant women with clinical symptoms of dengue infection who tested positive for dengue nonstructural protein 1-antigen or anti-dengue immunoglobulin M were included in the study. Clinical features and hematological and biochemical parameters were documented to assess their relationship with maternal and neonatal outcomes.

Results: Our study population consisted of 41 pregnant women. Most were multigravida (58.5\%). Dengue infection without warning signs was observed in $31.7 \%$ of the cases, while $53.7 \%$ had dengue infection with warning signs, and $14.6 \%$ had dengue shock syndrome/severe dengue infection. Most pregnancies were in the third trimester (78\%); five cases (12.2\%) suffered clinical complications. Preterm delivery was observed in seven cases (17.1\%). Most patients (75.6\%) decided to seek medical treatment at a critical phase of dengue infection (days 3-6).

Conclusion: The high incidence of associated morbidity and mortality should warrant clinicians in endemic regions to consider diagnoses of dengue infection when treating pregnant women with clinical signs and symptoms. Patients should be treated at centers with adequate resources for monitoring and emergency procedures. A more intensive study is needed in the future aiming to make a more comprehensive guideline.

Keywords: Dengue; Infections; Pregnancy; Maternal Outcome; Neonatal Outcome

Received: August 30, 2018, Revised: November 5, 2018, Accepted: December 4, 2018

${ }^{*}$ Corresponding Author: Tjokorda Gede Astawa Pemayun https://orcid.org/0000-0002-9936-9128

Tel: +62-8123843511, E-mail: mfmdenpasar@yahoo.com 


\section{INTRODUCTION}

Dengue infection is a disease endemic to Southeast Asia including Indonesia, and is associated with various complications. When the infection occurs during pregnancy, it potentially causes severe morbidity and mortality for both mother and the offspring. Managing a dengue infection occurring during a pregnancy is often difficult for clinicians due to a lack of global consensus for treatment, and inadequate guidelines consisting primarily of case series and expert opinions.

Dengue infection is a febrile illness caused by four closely related dengue virus serotypes (designated DENV-1, DENV-2, DENV-3, and DENV-4) of the genus Flavivirus and family Flaviviridae. According to the World Health Organization (WHO), dengue infection is classified as a dengue infection (with or without warning signs) or severe dengue infection. ${ }^{1)}$ Dengue infection occurs mostly in children; however, lately the incidence has been increasing in individuals of reproductive ages, including pregnant women. ${ }^{2)}$

Dengue infection is found in tropical and sub-tropical areas. According to WHO reports from 1968 to 2009, Indonesia had the highest number of individuals with dengue infection in Southeast Asia. ${ }^{3)}$ Based on the Indonesian health registry from 2010, most of the cases occurred in the province of Bali, followed by Jakarta, East Kalimantan, North Sulawesi, and Riau Islands. ${ }^{4)}$

Currently, there is no widely agreed consensus on the management of dengue infection during pregnancy. ${ }^{5)}$ Pregnant women are at a higher risk of more severe infection, and a higher mortality rate caused by dengue infection compared to general population. ${ }^{6}$ Several studies have indicated poor pregnancy outcomes for both mother and fetus. Gestational age and the phase of dengue infection at presentation are important factors in determining management and prognosis. ${ }^{7)}$ Few reports have been published regarding dengue infection during pregnancy in areas considered to be high-risk. A prospective study examining the outcomes of dengue infection during pregnancy in highly endemic countries, especially Indonesia, is important as the results may serve as the basis for decision-making and prognosis prediction regarding dengue infection "Therefore, we aimed to prospectively study the outcomes of dengue infection in pregnant women in Indonesia, during a period of outbreak from April 2016 to April 2017".

\section{METHODS}

We conducted a prospective observational study, undertaken at Sanglah General Hospital, Bali Indonesia from April 2016 to April 2017. Sanglah General Hospital is one of the largest tertiary-level hospitals in Indonesia, and serves almost one-third of the national population. We included 41 patients who tested positive for the nonstructural protein 1 (NS1) antigen or immunoglobulin M (IgM) anti-dengue in the study population. Positive test is determined as the presence of NS1 antigen or IgM in the serum. Only patients who presented in day 1-2 after the onset of fever were tested for NS1 antigen; all subjects were then tested for serum IgM and IgG on day 7. Demographic data, clinical and labo- ratory findings, and maternal and fetal outcomes were documented.

Disease severity was graded according to the WHO classification: (1) dengue infection with or without warning signs; and (2) severe dengue infection. According to the guidelines, a diagnosis of dengue infection should be considered for patients who live in or travel to areas where dengue is endemic, and who are presenting with a fever and two of the following criteria: nausea, vomiting, rash, aches and pains, a positive tourniquet test, leucopenia, or any other warning signs. Laboratory tests should then be used to confirm a dengue infection (NS1 antigen and IgM anti-dengue). Warning signs include abdominal pain or tenderness, persistent vomiting, clinical fluid accumulation, mucosal bleeding, lethargy, restlessness, liver enlargement of $>2 \mathrm{~cm}$, and increased hematocrit concurrent with a rapid decrease in platelet count. Criteria for severe dengue are: (1) severe plasma leakage leading to shock, in the case of dengue shock syndrome (DSS) and/or fluid accumulation with respiratory distress; (2) severe bleeding as evaluated by a clinician; (3) severe organ involvement (aspartate aminotransferase or alanine aminotransferase $\geq 1,000$, impaired consciousness, involvement of the heart and other organs). ${ }^{1)}$ Neonates with clinical signs and symptoms of dengue infection were tested for the presence of NS1 antigen during day 1-2 of fever, and were further confirmed using IgM and immunoglobulin G (IgG) testing. Vertical infection was diagnosed when clinical signs and symptoms of dengue infection were identified, supported by positive tests for the NS1 antigen, IgM, and IgG.

The presence of NS1 antigen in blood serum was tested on days 1-2 after the onset of fever. Patients presenting with signs and symptoms of dengue infection were tested, and blood samples $(3 \mathrm{~mL})$ were taken after obtaining informed consent from the patients. This study has been approved by the ethical board of Sanglah General Hospital. Dengue-specific antibodies were detected using the Panbio (Inverness Medical Innovations, Brisbane, Australia) Dengue Duo IgM and IgG Rapid strip test on serum samples taken between days 7-10 after the onset of fever. The test was based on a one-step sandwich format microplate enzyme immunoassay formulated to detect DENV and NS1 antigen in human serum samples. Serum samples of approximately $100 \mu \mathrm{L}$ each were assessed according to the manufacturer's instructions using an enzyme-linked immunosorbent assay (ELISA) Reader and ELISA Washer. The test results took around 2-3 hours. Quality control of the test was maintained according to the manufacturer's instructions. A dengue infection can be confirmed by a positive test for either anti-dengue IgM or the NS1 antigen. Anti-dengue IgG was also measured. An individual was diagnosed with a primary dengue infection only if they tested positive for IgM. A secondary dengue infection was indicated when tests for both anti-dengue IgM and IgG were positive.

Subjects were followed up till 6 weeks postpartum. Neonatal outcomes were also documented during the period. For neonates who tested positive for vertical transmission, the follow-up period was extended until 6 months to detect any long-term complications. Followup consisted of a clinical examination to assess the signs and symptoms of late dengue infection-associated complications, as well as the 
early growth and development of the neonates based on the Indonesian Neonatal Growth Chart. ${ }^{\text {8) }}$

\section{RESULTS}

In our study, we collected clinical data from 41 pregnant women diagnosed with dengue infection who were treated at Sanglah General Hospital. The information presented in Table 1 indicates that approximately $58.5 \%$ of the patients were multigravida and $41.5 \%$ were primigravida. Dengue infection without warning signs was observed in $31.7 \%$ of the cases, dengue infection with warning signs in $53.7 \%$ of the cases, and DSS/severe dengue infection in $14.6 \%$ of the cases. Most patients were in their third trimester of pregnancy (78\%), while $4.9 \%$ were in the first trimester and $17.1 \%$ in the second trimester. Thirty cases (73.2\%) tested positive for anti-dengue IgM, and 37 cases $(90.2 \%)$ tested positive for anti-dengue IgG, which indicated secondary dengue infection. Positive tests for the NS1 antigen were observed in 14 cases $(34.1 \%)$, while positive tests for both IgM and NSAg were observed in five cases (35.7\%). The presence of NS1 antigen was not tested in 27 patients, because they presented at a later phase of disease (after day 2 from the onset of fever).

Preterm delivery occurred in seven cases (17.1\%), while 34 cases (82.9\%) ultimately reached term pregnancy (Table 2). Five patients (12.2\%) underwent emergency cesarean section procedures for various complications (one patient with fetal distress, one with placental abruption, two with declining platelet counts, one with worsening pneumonia), eight patients (19.5\%) delivered normally, while 28 patients (68.3\%) experienced pregnancies that were conserved and were discharged without delivery.

Table 1. Characteristics of pregnant women with dengue infection in Sanglah General Hospital from April 2016-2017

\begin{tabular}{llc}
\hline \multicolumn{1}{c}{ Characteristic } & \multicolumn{1}{c}{ Category } & No. (\%) \\
\hline Gravida & Primigravida & $17(41.5)$ \\
& Multigravida & $24(58.5)$ \\
IgG Serology Test & Positive & $37(90.2)$ \\
Trimester & Negative & $4(9.8)$ \\
& 1st trimester & $2(4.9)$ \\
Pregnancy outcome & 2nd trimester & $7(17.1)$ \\
& 3rd trimester & $32(78)$ \\
NS1 test & Term labor & $34(82.9)$ \\
& Preterm labor & $7(17.1)$ \\
Serologi IgM & Positive & $14(34.1)$ \\
& Not checked & $27(65.9)$ \\
Clinical diagnosis & Positive & $30(73.2)$ \\
& Negative & $11(26.8)$ \\
& Dl without WS & $13(31.7)$ \\
Mode of delivery & Dl with WS & $22(53.7)$ \\
& Severe dengue & $6(14.6)$ \\
& Cesarean section & $5(12.2)$ \\
& Normal labor & $8(19.5)$ \\
& Conservative & $28(68.3)$ \\
\hline
\end{tabular}

Ig, immunoglobulin; NS1, nonstructural protein 1; DI, Dengue infection; WS, warning sign.
Two cases of early pregnancy with dengue infection were reported; one case $(2.4 \%)$ had a spontaneous abortion and the other $(2.4 \%)$ progressed to term. Three babies (7.3\%) were born with asphyxia, while one case $(2.4 \%)$ was diagnosed with oligohydramnios and dengue encephalitis (vertical infection). None of the neonates showed signs of congenital anomaly.

We observed two cases (4.9\%) with postpartum hemorrhage, two cases $(4.87 \%)$ with at-term intrauterine fetal death (IUFD), one case (2.4\%) with placental abruption, and 12 cases (29.3\%) with severe thrombocytopenia. Maternal and neonatal complications were more common in individuals with secondary infections (10 cases of severe thrombocytopenia, one case of oligohydramnios, five cases of severe dengue infection, one case of dengue encephalitis, five cases of maternal death, and two cases of IUFD). Most patients (75.6\%) sought medical attention at a critical phase of dengue infection (days 3-6), but only 13 patients (31.7\%) needed intensive care. One maternal death occurred in the first trimester and five deaths (12.2\%) occurred in the third trimester. A comparison of the results of our study with that of Basurko et al. ${ }^{9)}$ and Agrawal et al. ${ }^{7)}$ is presented in Table 3.

Conservative medical and obstetrical management were the treatments of choice. Only women who went into labor required a platelet transfusion, depending on the platelet count. Outcomes are also correlated with the gestational age. ${ }^{7)}$

In the absence of maternal-fetal complications, decision to deliver the baby is not mandatory. In this setting, we prefer a supportive ther-

Table 2. Maternal and neonatal complications

\begin{tabular}{lc}
\hline \multicolumn{1}{c}{ Variable } & No. $(\%)$ \\
\hline Preterm labor & $7(17.1)$ \\
Postpartum hemorrhage & $2(4.9)$ \\
Intrauterine fetal death & $2(4.9)$ \\
Abortion & $1(2.4)$ \\
Asphyxia & $3(7.3)$ \\
Oligohydramnios & $1(2.4)$ \\
Dengue encephalitis & $1(2.4)$ \\
Day fever & $8(19.6)$ \\
1-2 (febrile) & $31(75.6)$ \\
3-6 (critical) & $2(4.8)$ \\
7-10 (recovery) & \\
Intensive care unit & $13(31.7)$ \\
Severe thrombocytopenia & $12(29.3)$ \\
Maternal mortality & $6(14.6)$ \\
\hline
\end{tabular}

Table 3. Comparison with other studies

\begin{tabular}{lccc}
\hline \multicolumn{1}{c}{ Variable } & Basurko et al. ${ }^{9)}$ & Agrawal et al. ${ }^{7)}$ & Our study \\
\hline Premature labor & 41 & 52 & 17.1 \\
Oligohydramnios & 46 & 52 & 2.4 \\
Antepartum hemorrhage & 9.3 & 32 & 2.4 \\
Postpartum hemorrhage & 10 & 36 & 4.9 \\
Abortions & 3.8 & 16 & 2.4 \\
Intrauterine fetal death & 7.5 & 16 & 4.9 \\
Neonatal death & 1.9 & 18 & 0
\end{tabular}

Values are presented as \%. 
apy as the treatment of choice, and only those who are in labor may require platelet transfusion.

\section{DISCUSSION}

During the most recent outbreak of dengue infection in Bali, Indonesia, we identified 41 seropositive cases, resulting in four cases of maternal mortality. We believe that it is important to consider a diagnosis of dengue infection in all pregnant women presenting with acute febrile illnesses, especially in endemic regions. Maternal and neonatal complications are potentially fatal, and appropriate treatments including fluid resuscitation and close monitoring have to be carried out by clinicians. $^{6,9)}$

Similar to other studies, we found that most patients sought care during the third trimester of pregnancy. ${ }^{10,11)}$ The physiological increase in intravascular volume in the third trimester would bias the identification of plasma leakage syndrome, thus causing an underestimation of the burden of dengue infection. ${ }^{12,13)}$ Our study found that secondary infections were more common than primary infections (90.2\% versus $9.8 \%$, respectively). This pattern is similar to that observed in nonpregnant adults. ${ }^{12)}$ As aforementioned, the incidence of dengue infection in Indonesia was the highest globally. From 1993 to 1998, the highest number of cases occurred in individuals $<15$ years, while from 1999 to 2009, dengue infection was mostly observed in individuals aged $\geq 15$ years. ${ }^{14)}$ The number of cases in all the age groups has increased over the last 20 years and peaked in the last decade. ${ }^{15,16)}$

Adverse fetal outcomes may be attributed to the effects on placental circulation that are associated with endothelial damage and increased vascular permeability, which lead to plasma leakage. ${ }^{17)}$ In a prospective cohort, Tan et al. ${ }^{14)}$ reported vertical transmission in one out of 63 cases (1.6\%). Basurko et al. ${ }^{9)}$ reported a $5.6 \%$ rate of maternal-fetal transmission. Two studies from Cuba and French Guiana reported vertical dengue transmission in four out of $59(6.8 \%)$ neonates and two out of 19 (10.5\%) neonates, respectively. ${ }^{15,16)}$ However, different results were observed in a North Indian study, which reported no diagnoses of vertical infection in eight pregnancies with dengue infection. ${ }^{17)}$ In this study, we found only one case of vertical transmission (2.4\%). Unfortunately, this number does not represent the actual incidence of vertical transmission, because a polymerase chain reaction examination, known as the diagnostic gold standard, was not routinely performed due to financial constraints. We believe that the rate of maternal-fetal transmission is higher in general population outside the study subjects and should be taken into consideration when treating neonates.

It has been suggested that the dengue-specific IgG transferred from mother to fetus in utero plays a key role in the pathogenesis of neonatal effects. ${ }^{18)}$ Many conditions such as fever, petechial rash, thrombocytopenia, leucopenia, elevated liver enzymes, hepatomegaly, pleural effusion, premature birth, fetal malformations, miscarriages, and low birth weight have been reported. In our study, the symptoms of dengue infection in the sole neonate who experienced vertical transmis- sion were fever, thrombocytopenia, and leucopenia. The neonate did not experience severe symptoms and was discharged in good condition. A systematic review including 38 severe cases registered in French Guiana reported five IUFDs (13.1\%). ${ }^{15)}$ In our study, there were six cases with severe dengue infection, of which two cases $(4.87 \%)$ resulted in IUFD in the third trimester. This number is lower than what is observed in other studies. The clinical stage at presentation, gestational age, presence of adequate facilities, and awareness of the healthcare provider are important factors for patient prognosis. To prevent adverse perinatal outcomes, a clinical diagnosis has to be made rapidly, and patients should be treated in centers with adequate monitoring resources, and the facilities to perform 'green code' cesarean section procedures.

A study with a 5-year follow-up period for four neonates in Cuba who had vertical dengue infection found no long-term sequelae in all the patients studied. ${ }^{16)}$ Another study with a year of follow-up with three vertically infected neonates reported similar results. ${ }^{19)}$ In our study, the affected neonate was treated in the hospital for 10 days without the need for platelet transfusion, and was discharged in good condition. During the 6-month follow-up period, we found no evidence of morbidity, or any disruption in the development and growth of the affected neonate.

Severe thrombocytopenia was diagnosed in 12 cases (29.3\%). Among six maternal deaths reported in this study, five patients (83.3\%) experienced severe thrombocytopenia, whereas all cases of IUFDs experienced severe maternal thrombocytopenia. This would suggest that severe thrombocytopenia is associated with maternal mortality and IUFD, although further studies involving larger sample sizes are required to determine the correlation between these variables.

We documented six cases (14.6\%) with DSS, of which five cases (83.3\%) resulted in maternal deaths. Another case of maternal death occurred in a patient with dengue infection with warning sign. DSS is potentially fatal and may progress to multi-organ failure, including acute respiratory distress syndrome, as described by Lum et al. ${ }^{20)}$ Studies have indicated the presence of an increased rate of cesarean sections as well as increased risk of hemorrhage, preeclampsia, miscarriages, fetal anomalies, low birth weight, and premature births in pregnant women with dengue infection. Severe dengue infection has been associated with maternal deaths, with fatality rates ranging from $2.9 \%-$ 22\%. ${ }^{6)}$ A study in Rio de Janeiro in 2007 showed that pregnant women with dengue infection were 3-4 times more likely to develop severe dengue (odds ratio, 3.38; 95\% confidence interval, 2.10-5.42). Mortality rates among pregnant women were also higher than in non-pregnant women (3.0\% versus $1.1 \%$ ). The high levels of morbidity and mortality attributed to severe dengue infection should warrant awareness among clinicians in treating dengue infection in pregnancy. Adequate monitoring and hydration status should be priorities, and platelet transfusion should always be prepared. ${ }^{1)}$ These measures are vital as pregnant women are prone to sudden haemodynamic deterioration; Physiologic changes in pregnancy may mask the real extent of infection and make it hard for physicians to determine prognosis. 
It is well-documented that sequential infection with different dengue serotypes predisposes individuals to more severe forms of the disease. This is explained by the enhancement of the cross-reactive cascade of amplified non-neutralizing heterologous antibodies, cytokines (e.g., interferon-gamma produced by specific T-cells), and complement activation, further causing endothelial dysfunction, platelet destruction, and consumptive coagulopathy. ${ }^{21,22)}$ In this study, we found that maternal and neonatal complications were more common in secondary infections (severe thrombocytopenia, severe dengue infection, dengue encephalitis, maternal death, oligohydramnios, and IUFD). These findings may describe the adverse potential of secondary infection in our population. This data may be used to counsel patients regarding prognosis.

Thirty-one cases (75.6\%) sought care during the critical phase (days 3-6), of which six cases (19.4\%) experienced maternal deaths. We suggest that every pregnant woman presenting with a recent onset of high-grade fever in endemic areas, especially during the epidemic period, should be suspected to have contracted dengue until proven otherwise. A delay in diagnosis is quite common due to similar clinical and laboratory findings with other conditions associated with pregnancy, such as HELLP (hemolysis, elevated liver enzymes, and low platelet count) syndrome, pneumonia, pulmonary embolism, conditions that cause vaginal bleeding, and various infectious diseases. ${ }^{1)}$

In this study, a cesarean section was performed for five patients due to maternal or fetal compromise, while eight cases had normal deliveries, and 28 pregnancies were conserved. Further, 13 cases $(31.7 \%)$ needed intensive care.

The findings of our study may represent the burden of dengue in endemic countries. Furthermore, this study was conducted during a dengue outbreak in Indonesia, and all cases were obtained during this period. To the best of our knowledge, this is the first study to present data on dengue infection occurring during pregnancy during an outbreak. The findings of our study are particularly important for endemic countries when building a treatment protocol, since we describe the impact of a dengue outbreak on maternal and neonatal health. Based on the results of our study, we conclude that the incidence of dengue infection during pregnancy may be higher than expected. In endemic areas, concerns regarding dengue infection should be raised during epidemics. Pregnancies complicated by dengue infection require close monitoring to avoid further maternal and fetal complications.

\section{CONFLICTS OF INTEREST}

No potential conflict of interest relevant to this article was reported.

\section{ACKNOWLEDGMENTS}

We would like to thank the director of Sanglah General Hospital and the Head of the Obstetrics and Gynecologic department for their support in this research study.

\section{ORCID}

Ryan Saktika Mulyana: https://orcid.org/0000-0002-6683-5858

Evert Solomon Pangkahila: https://orcid.org/0000-0003-1434-9097

Tjokorda Gede Astawa Pemayun: https://orcid.org/0000-0002-9936-9128

\section{REFERENCES}

1. See Lum LC, Guzman MG, Martinez E, Tan LH, Hung NT. Handbook for clinical management of dengue [Internet]. Geneva: World Health Oganization; 2012 [cited 2017 Dec 20]. Available from: http://whqlibdoc.who.int/publications/2009/9789241547871_eng.pdf.

2. Paixao ES, Teixeira MG, Costa MDCN, Rodrigues LC. Dengue during pregnancy and adverse fetal outcomes: a systematic review and metaanalysis. Lancet Infect Dis 2016;16:857-65.

3. Achmadi UF. Manajemen demam berdarah berbasis wilayah. Bul Jendela Epidemiol 2010;2:15-20.

4. Pangribowo S, Tryadi A, Indah IS. Demam berdarah dengue di Indonesia tahun 1968-2009. Bul Jendela Epidemiol 2010;(2):1-14.

5. Feitoza HAC, Koifman S, Koifman RJ, Saraceni V. Dengue infection during pregnancy and adverse maternal, fetal, and infant health outcomes in Rio Branco, Acre State, Brazil, 2007-2012. Cad Saude Publica 2017;33:e00178915.

6. Machado CR, Machado ES, Rohloff RD, Azevedo M, Campos DP, de Oliveira RB, et al. Is pregnancy associated with severe dengue?: a review of data from the Rio de Janeiro surveillance information system. PLoS Negl Trop Dis 2013;7:e2217.

7. Agrawal P, Garg R, Srivastava S, Verma U, Rani R. Pregnancy outcome in women with dengue infection in Northern India. Indian J Clin Pract 2014;24:1053-6.

8. Menteri Kesehatan Republik Indonesia. Regulation of the Ministry of Health of the Republic of Indonesia no 155 of 2010 concerning use of the card for toddlers growth monitoring. Jakarta: Menteri Kesehatan Republik Indonesia; 2010.

9. Basurko C, Carles G, Youssef M, Guindi WE. Maternal and fetal consequences of dengue fever during pregnancy. Eur J Obstet Gynecol Reprod Biol 2009;147:29-32.

10. Pavanaganga A, Sailakshmi MPA, Rekha BR, Nagarathnamma R. Dengue fever during pregnancy: maternal and fetal complications. J South Asian Feder Obst Gynae 2017;9:88-91.

11. Waduge R, Malavige GN, Pradeepan M, Wijeyaratne CN, Fernando S, Seneviratne SL. Dengue infections during pregnancy: a case series from Sri Lanka and review of the literature. J Clin Virol 2006;37:27-33.

12. Malavige GN, Velathanthiri VG, Wijewickrama ES, Fernando S, Jayaratne SD, Aaskov J, et al. Patterns of disease among adults hospitalized with dengue infections. QJM 2006;99:299-305.

13. Iyngkaran N, Yadav M, Sinniah M. Augmented inflammatory cytokines in primary dengue infection progressing to shock. Singapore Med J 1995;36:218-21.

14. Tan PC, Rajasingam G, Devi S, Omar SZ. Dengue infection in pregnancy: prevalence, vertical transmission, and pregnancy outcome. Obstet Gynecol 2008;111:1111-7.

15. Carles G, Talarmin A, Peneau C, Bertsch M. Dengue fever and pregnancy: a study of 38 cases in french Guiana. J Gynecol Obstet Biol Re- 
prod (Paris) 2000;29:758-62.

16. Fernandez R, Rodriguez T, Borbonet F, Vazquez S, Guzman MG, Kouri G. Study of the relationship dengue-pregnancy in a group of Cubanmothers. Rev Cubana Med Trop 1994;46:76-8.

17. Malhotra N, Chanana C, Kumar S. Dengue infection in pregnancy. Int J Gynaecol Obstet 2006;94:131-2.

18. Watanaveeradej V, Endy TP, Samakoses R, Kerdpanich A, Simasathien S, Polprasert N, et al. Transplacentally transferred maternal-infant antibodies to dengue virus. Am J Trop Med Hyg 2003;69:123-8.
Ryan Saktika Mulyana, et al. • Dengue Infection in Pregnancy in Bali

19. Phongsamart W, Yoksan S, Vanaprapa N, Chokephaibulkit K. Dengue virus infection in late pregnancy and transmission to the infants. Pediatr Infect Dis J 2008;27:500-4.

20. Lum LC, Thong MK, Cheah YK, Lam SK. Dengue-associated adult respiratory distress syndrome. Ann Trop Paediatr 1995;15:335-9.

21. Gibbons RV, Vaughn DW. Dengue: an escalating problem. BMJ 2002; 324:1563-6.

22. Guzman MG, Vazquez S, Kouri G. Dengue: where are we today? Malays J Med Sci 2009;16:4-11. 